\title{
On determining leading coalitions in supply chains: methodology and application
}

\author{
Kidd, Martin Philip; Borm, Peter
}

Link to article, DOI:

10.2139/ssrn.3805728

Publication date:

2021

Document Version

Publisher's PDF, also known as Version of record

Link back to DTU Orbit

Citation (APA):

Kidd, M. P., \& Borm, P. (2021). On determining leading coalitions in supply chains: methodology and application. Tilburg University. Center for Economic Research. Discussion Paper (Online) No. 2021-009 https://doi.org/10.2139/ssrn.3805728

\section{General rights}

Copyright and moral rights for the publications made accessible in the public portal are retained by the authors and/or other copyright owners and it is a condition of accessing publications that users recognise and abide by the legal requirements associated with these rights.

- Users may download and print one copy of any publication from the public portal for the purpose of private study or research.

- You may not further distribute the material or use it for any profit-making activity or commercial gain

- You may freely distribute the URL identifying the publication in the public portal

If you believe that this document breaches copyright please contact us providing details, and we will remove access to the work immediately and investigate your claim. 
No. 2021-009

\title{
ON DETERMINING LEADING COALITIONS IN SUPPLY CHAINS: METHODOLOGY AND APPLICATION
}

\author{
By
}

Martin P. Kidd, Peter Borm

16 March 2021

ISSN 0924-7815

ISSN 2213-9532 


\title{
On determining leading coalitions in supply chains: methodology and application
}

\author{
Martin P Kidd ${ }^{1}$, Peter Borm ${ }^{2}$ \\ ${ }^{1}$ Department of Technology, Management and Economics, \\ Technical University of Denmark, Lyngby, Denmark \\ ${ }^{2}$ CentER and Department of Econometrics and Operations Research, \\ Tilburg University, The Netherlands
}

\begin{abstract}
Supply chain collaboration has the potential to address issues of inefficiency and lack of coordination that often drives down the competitiveness of supply chains. However, collaboration in supply chains is difficult due to ever increasing complexity and conflicting objectives prevalent in supply chains, and a growing challenge is to be able to identify the optimal configuration of collaboration that benefits the supply chain as a whole. The inherent complexity of supply chains has lead to the emergence of so-called orchestrators to help coordinate supply chains and enable collaboration. In this paper we address a specific problem that an orchestrator might face: given a set of potential coalitions that could form in a given supply chain, together with the means by which collaborative benefits should be allocated, which coalition should form? We propose a new methodology based on what we call leading coalitions to address this coalition selection problem, which borrows concepts from cooperative game theory to ensure stability and fairness of the final selection. The proposed methodology is applied to linear production processes where agents collaborate by pooling their resources, and a computational study is performed on a large set of instances in order to gain insight into the working of the methodology.
\end{abstract}

Keywords: Supply chain collaboration; Cooperative bargaining; Nucleolus selections; Linear production processes

\section{Introduction}

In order to be responsive to dynamic market needs at lower costs, an indispensable tool for any company operating within a supply chain is collaboration with other supply chain members (Christopher, 2011; Soosay and Hyland, 2015). Companies have realized that there is much to gain in terms of competitive advantage by focussing efforts towards end-to-end coordination and relationship management, and by taking a system-wide perspective in supply chain decision-making and planning (Simchi-Levi et al., 2008). Supply chain collaboration comes in many different forms. Vertical collaboration consists of supply chain members from different stages or tiers in the supply chain, and some examples include the sharing of information (e.g. forecasts) (Raweewan and Ferrell, 2018), coordination contracts (Schneeweiss et al., 2004), and joint decision making (e.g. collaborative planning) (Aviv, 2001; Kilger et al., 2015). A number of vertical collaboration initiatives have seen considerable success in practice, such as vendormanaged inventory (VMI) and collaborative planning, forecasting and replenishment (CPFR) (Nimmy et al., 2019). On the other hand, horizontal collaboration consists of supply chain members from the same stage or tier in the supply chain, and examples include inventory pooling (Dror and Hartman, 2011) and collaborative logistics (Pan et al., 2019; Ferrell et al., 2020). Collaborative logistics in particular has seen a number of successful implementations in industry, especially in Europe (Verstrepen et al., 2009). 
Despite its perceived importance and the success in some industries, supply chain collaboration has been difficult to implement for many companies due to issues such as mis-alignment of goals, opportunistic behaviour, and lack of trust (Fawcett et al., 2012). Moreover, Soosay and Hyland (2015) emphasizes that there is a need for taking a multi-tier perspective to supply chain collaboration, where suppliers, manufacturers, distributors, retailers, etc., are all working in unison towards a more competitive supply chain. However, supply chains are complex structures that often have a global span, and supply chain collaboration from this holistic perspective would require large coalitions consisting of many different companies throughout a supply chain. There are a number of different factors that prohibit the formation of large coalitions, including leadership issues, the cost of establishing a common information sharing infrastructure, and risk of antitrust violations (Basso et al., 2019). Another factor that presents itself as an impediment to the formation of large coalitions is cooperation complexity, which is driven by the number of agents involved (e.g. companies and business functions), the geographical and competence heterogeneity of the agents involved, and direct competition in the case of horizontal collaboration (Schmoltzi and Wallenburg, 2012). Stevens and Johnson (2016) suggest that complexity is leading supply chains towards a new era of integration characterized by "collaborative supply chain clusters" which are smaller networks within the wider supply chain where collaboration and integration can be more easily managed. The result is thus a clustered form of collaboration where smaller coalitions are established among different members of the supply chain, and where not all potential coalitions can form simultaneously. When there are many different coalitions that can potentially form, a natural question that arises from a holistic supply chain perspective is which coalitions should form. This is the central question addressed in this paper.

An example of this kind of clustered collaboration can be found in a case study by Frisk et al. (2010), who studies collaboration amongst eight forestry companies in southern Sweden. Successful coalitions will enter into wood bartering or backhauling agreements when it comes to the transportation of logs from harvest areas to pulp- and sawmills. Benefits include reduced transportation costs and higher utilization of trucks. Although ideally all eight companies would collaborate, there are a number of difficulties that prohibit this, including the fact that planning and decision making become more complex as more companies are involved and that care should be taken to not violate antitrust laws. In particular, when it comes to wood bartering, it is rare that more than two companies collaborate (Frisk et al., 2010). With these restrictions in mind, a follow-up study by Guajardo and Rönnqvist (2015) study the problem of which coalitions should form. In the same paper the authors apply the same model to a second case study based on spare part inventory pooling in the oil industry.

In both the above mentioned two case studies presented by Guajardo and Rönnqvist (2015), an external third party was the primary decision maker addressing the question of which coalitions should form. In the forestry case, for example, it was a consulting team responsible for the analysis and recommendation of how collaboration should be structured among the companies. This relates closely to a concept that has recently emerged to address the increasing complexity of supply chains, as well as the various barriers to collaboration mentioned above, namely that of an orchestrator, i.e. a neutral external party whose main task is to coordinate a supply chain. According to Bitran et al. (2007) there is a growing need for neutral third parties to coordinate supply chains and align incentives, and Stephens (2006) finds that supply chain collaboration is more likely to take place if it is coordinated by an external party. In general, the essential task of an orchestrator is to align the objectives of different independent actors within a supply chain (Fulconis and Paché, 2018) and to enable collaboration (Zacharia et al., 2011). An example of this is a fourth party logistics (4PL) provider, which can be defined as an independent integrator that offer services related to the design, management and coordination of complex supply chains (Win, 2008). Although 4PL providers may offer various services such as analytics, planning and monitoring, its fundamental purpose is to foster end-to-end supply chain integration and relationship management among supply chain partners (Schramm et al., 2019). Moreover, 4PL 
service providers have the potential to overcome the impediments and barriers to supply chain collaboration mentioned above (Hingley et al., 2011). An important capability of a supply chain orchestrator is therefore to be able to identify the most promising coalitions within a supply chain network, and to address the question mentioned above of which coalitions should form. In particular, its visibility across the supply chain puts it in the ideal position for this task.

In this paper we consider a new supply chain collaboration model which we call a cooperative selection situation, where an orchestrator needs to decide which among a given set of coalitions in a supply chain should form. We assume that potential coalitions have already been identified, either by supply chain members themselves or by the orchestrator, and that agreements have already been made as to how the benefits of collaboration may be allocated should any of these potential coalitions form. Given this, a selection then consists of a coalition together with one of its proposed allocation vectors. We therefore do not directly address the question of how benefits are to be allocated, as is the case in many studies of supply chain collaboration, but instead we assume this as given and focus more on the problem that multiple possible, potentially conflicting coalitions have been proposed and that a decision needs to be made as to which one to select. We propose a selection model that takes the stability and fairness of the final outcome into account, and our model is based on various concepts borrowed from cooperative game theory, in particular on the bargaining set (Aumann and Maschler, 1961; Maschler, 1992; Sudhölter and Potters, 2001). Stability and fairness are important since collaboration requires agreement from all members of a coalition for it to be successful, and in order for an orchestrator to foster collaboration across an entire supply chain, members need to trust that the orchestrator is capable of establishing successful coalitions.

In order to address stability, we consider that a selection may be blocked by a subset of the agents in the selected coalition if they can all strictly do better if another selection were made. This gives rise to blocked selections, and selections that are not blocked we call leading selections and their corresponding coalitions are called leading coalitions. Fairness is taken into account by calculating an excess for each potential coalition with respect to a specific selection, which is the difference between its value and what its members are allocated in total in the selection. Our model then narrows the leading selections down to the ones for which excesses are minimized lexicographically. In order to illustrate and gain some insights into the working of our proposed methodology, we apply our model to a situation where multiple manufacturers have access to a linear production process and can collaborate by pooling their resources. Our focus will be on a context-specific solution concept, the Owen set, as introduced by Owen (1975) and axiomatically characterized by Van Gellekom et al. (2000).

We would like to stress that the emphasis of this paper is on the methodology, and as far as we are aware, the approach we follow is new. The most closely related literature is on coalition formation. The coalition formation literature is mostly concerned with partitioning agents into disjoint coalitions and determining how to distribute the worth of each coalition; see for example the works of Elomri et al. (2012) and Guajardo and Rönnqvist (2015), and the references therein. However, our case is different, since we assume only one coalition can form, and only from a restricted set of coalitions, and that the means of distributing the worth of this one coalition has already been decided upon.

The rest of the paper is organized as follows. The proposed methodology is formally introduced in Section 2. In Section 3 the application of the methodology to linear production processes is described and illustrated through the use of a number of examples. In Section 4 computational experiments are conducted on a large set of randomly generated instances of linear production processes in order to generate further insights into the working of the methodology, and the section closes a summary of the main findings. Finally, Section 5 concludes the paper. 


\section{The model}

We consider a supply chain network that comprises a finite set $N=\{1,2, \ldots,|N|\}$ of agents who represent the main decision makers in the supply chain. Depending on the specific case, these agents could, for example, represent different retailers, third-party logistics service providers, manufacturers, suppliers, and so on. In our example application in Section 3, the agents will correspond to a set of manufacturers that produce and sell a similar product.

We assume that the agents can collaborate in various ways, e.g. by sharing resources, sharing information, coordinated planning, and so on, and that this done by forming coalitions (subsets of agents). Importantly, we assume that there are costs and/or restrictions to collaboration, so that not any coalition can necessarily form. Costs can be related to administration, IT infrastructure changes, negotiations, etc., that are needed for collaboration to be able to take place. Restrictions can come in the form of antitrust regulations, conflicts of interest between different agents, or simply a lack of trust among certain agents. We assume that whatever the costs and restrictions are, they have been used to identify a set of coalitions that are deemed feasible. We are thus provided with a set $F \subseteq 2^{N}$ of feasible leading coalitions, where $|S|>1$ for any $S \in F$.

Along with each feasible leading coalition, we assume that the means of allocating the benefits of collaboration, were the coalition to form, has been agreed upon. Typically this would be done using techniques from cooperative game theory. More specifically, in games with transferable utility (TU) a coalition is associated with a "worth" expressed by a single number which represents a monetary payoff (e.g. profits) that can distributed among agents in any way. Most concepts from cooperative game theory address the problem of how the worth of a coalition should be distributed, the most well-known concepts being the core, the Shapley value (Shapley, 1953) and the nucleolus (Schmeidler, 1969). Given a feasible leading coalition, an agreement could therefore be that the Shapley value or nucleolus will be used to allocate the coalitional value. In this case, a unique allocation to each agent has already been decided upon, should the coalition form. However, we assume that this is not necessarily the case, and that an agreement could also have been made that, for example, an element of the core is to be used. In this case multiple possible allocations might exist, and there is some freedom to allow other criteria to play a role in defining the final allocation. We also assume to be given what agents earn if they do not participate in a coalition, i.e. if they act in isolation.

Each coalition $S \in F$ and each $S=\{i\}, i \in N$, is therefore associated with a set $V(S) \subseteq \mathbb{R}^{N}$ of payoff vectors, where each $x \in V(S)$ specifies a payoff $x_{i}$ to agent $i \in N$, where $x_{i}=0$ if $i \notin S$. A payoff to an agent can represent, for example, part of the revenue or profit generated by the coalition, as it will in our example application in Section 3. In collaborative logistics and supply chain planning, on the other hand, the main benefits of collaboration is usually in the reduction of logistical costs . In such a case the "payoff" to an agent usually represents an allocation of the total coalition costs, which in our case would correspond to a negative payoff. Depending on how the payoff vectors are defined in such studies, it could also represent a share of the cost savings generated by the coalition (Audy et al., 2012). In either case, we assume that agents want to maximize their payoffs.

We assume that each payoff set $V(S) \subseteq \mathbb{R}^{N}$ for any $S \in F$ or $S=\{i\}, i \in N$, satisfies two conditions. First of all, $V(S)$ is either finite or a convex polytope. Secondly, there is a number $v(S) \in \mathbb{R}$ such that $v(S)=\sum_{i \in S} x_{i}$ for any $x \in V(S)$, i.e. all payoff vectors efficiently distribute the benefits of collaboration. Note that the latter condition implies that $|V(S)|=1$ if $S=\{i\}, i \in N$, i.e. an agent acting in isolation receives a unique payoff.

A triple $(N, F, V)$, with $N, F$, and $V$ defined as discussed above, we call a cooperative selection situation. The problem is to identify which coalition in $F$ should form, given that the payoffs to agents in the coalition will be defined by $V$. More formally, the problem is to identify a selection, which is defined as a pair $(S, x) \in F \times \mathbb{R}^{N}$ such that $x_{S}=y_{S}$ for some $y \in V(S)$ and $x_{N \backslash S}=(v(\{i\}))_{i \in N \backslash S}$. We thus assume as a final restriction that only one coalition from $F$ 
can be selected. The forming of a specific coalition and the execution of the collaborative efforts required can be seen as a project to be undertaken by an orchestrator with capacity to do only one such a project at a time.

We propose the following procedure for identifying a selection. We start by following an approach similar to the one behind the bargaining set in cooperative game theory (Aumann and Maschler, 1961) that aims at obtaining a stable agreement among the agents by assuming they might have legitimate objections to certain outcomes. We say that a selection $(S, x)$ is blocked by a selection $(T, y)$ if $S \cap T \neq \varnothing, y_{i}>x_{i}$ for all $i \in S \cap T$ and $y_{i}>v(\{i\})$ for all $i \in T \backslash S$. In other words, $(S, x)$ is blocked if some of the members of $S$ find that they can, through another selection, offer strictly higher payoffs to agents outside $S$ while securing strictly higher payoffs for themselves. We are then particularly interested in selections that are not blocked by any other selection. We call such selections leading and denote by $\mathscr{L}(N, F, V)$ the set of all leading selections. Moreover, a coalition $S \in F$ is called leading if there exists a leading selection $(S, x)$, and let $L(N, F, V)$ denote the set of leading coalitions. The set of leading allocations is given by

$$
U(N, F, V)=\left\{x \in \mathbb{R}^{N} \mid(S, x) \in \mathscr{L} \text { for some } S \in F\right\} .
$$

In case there can be no confusion about the underlying cooperative selection situation $(N, F, V)$, we write $\mathscr{L}$ instead of $\mathscr{L}(N, F, V), L$ instead of $L(N, F, V)$ and $U$ instead of $U(N, F, V)$.

Whereas a leading selection ensures that no group of agents has a legitimate objection, the next step we propose aims to minimize dissatisfaction with a given leading selection in the same way the nucleolus in cooperative game theory does (Schmeidler, 1969; Potters and Tijs, 1992). We therefore define the excess of a coalition $T \in F$ with respect to a payoff vector $x \in \mathbb{R}^{N}$ as $E(T, x)=v(T)-\sum_{i \in T} x_{i}$. In other words, the excess of a coalition represents the increase in payoff for the coalition as a whole if it were to be selected. These excesses can be used to measure the overall satisfaction with a given payoff vector $x \in \mathbb{R}^{N}$. As we shall see, the outcome depends on which coalitions' excesses are taken into consideration. We will consider two approaches. In the first, we consider the excesses of all feasible leading coalitions, and in the second, we will consider only the excesses of leading coalitions. For any $G=F$ or $G=L$ and $x \in U(N, F, V)$, let $\theta(G, x)$ represent the vector of excesses with respect to $x$ of all coalitions in $G$ ordered from high to low. Then we define a generalized nucleolus given by

$$
\mathscr{N}^{G}(N, F, V)=\{(S, x) \in \mathscr{L}(N, F, V) \mid \theta(G, x) \preceq \text { lex } \theta(G, y) \text { for all } y \in U(N, F, V)\}
$$

where $\preceq_{\text {lex }}$ denotes the lexicographical order on $\mathbb{R}^{|G|}$. In other words, this set contains those leading selections, henceforth referred to as nucleolus selections, whose allocations have lexicographically minimal excesses for coalitions in $G$. In case there can be no confusion about the underlying cooperative selection situation $(N, F, V)$, we write $\mathscr{N}^{G}$ instead of $\mathscr{N}^{G}(N, F, V)$.

As mentioned above, we will consider two nucleoli, namely $\mathscr{N}^{F}$ and $\mathscr{N}^{L}$, and try to shed some light on the effect of using one or the other. The difference between these two approaches is that when using $\mathscr{N}^{L}$ the dissatisfaction of coalitions that aren't leading are disregarded, while when using $\mathscr{N}^{F}$ this is not the case.

The following example is used to illustrate the full model.

Example 1 Consider a cooperative selection situation $(N, F, V)$ with three agents where only coalitions of at most two agents can form, i.e. $N=\{1,2,3\}$ and $F=\{\{1,2\},\{1,3\},\{2,3\}\}$. The payoffs represent collaborative gains such that $v(\{i\})=0$ for all $i \in N$. The total payoffs to the coalitions are $v(\{1,2\})=3, v(\{1,3\})=4$ and $v(\{2,2\})=5$, and each coalition has identified two acceptable payoff vectors, given by

$$
V(S)= \begin{cases}\{(0,3,0),(1,2,0)\} & \text { if } S=\{1,2\}, \\ \{(0,0,4),(1,0,3)\} & \text { if } S=\{1,3\}, \\ \{(0,2,3),(0,4,1)\} & \text { if } S=\{2,3\}\end{cases}
$$


An example of a selection is $(\{1,2\},(0,3,0))$. As it so happens, this selection is blocked since agent 1 can do strictly better in another selection, namely $(\{1,3\},(1,0,3))$, where agent 3 also does better compared to acting alone.

Whenever $V(S)$ is finite for all $S \in F$, we can associate a blocking graph to the cooperative selection situation, which contains a vertex associated with each selection and an arc directed from $(S, x)$ to $(T, y)$ if $(S, x)$ is blocked by $(T, y)$. By definition, the sinks of this graph, if any, correspond to the leading coalitions. The blocking graph for this example is shown in Figure 1. The sinks of this graph, and thus the leading selections, are $(\{1,3\},(1,0,3))$ and $(\{2,3\},(0,2,3))$.

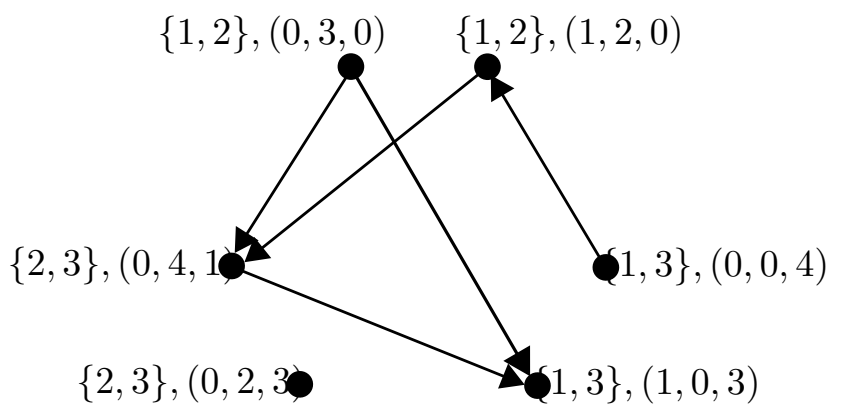

Figure 1: Blocking graph of the cooperative selection situation in Example 1.

The set of leading selections, leading coalitions and leading allocations is therefore given by

$$
\begin{gathered}
\mathscr{L}=\{(\{1,3\},(1,0,3)),(\{2,3\},(0,2,3))\}, \\
L=\{\{1,3\},\{2,3\}\}, \text { and } \\
U=\{(1,0,3),(0,2,3)\} .
\end{gathered}
$$

Next we consider the excesses of all coalitions with respect to the two leading allocations. For example, for the allocation $x=(1,0,3)$ the excess of the coalition $\{1,2\}$ is

$$
E(\{1,2\}, x)=v(\{1,2\})-x_{1}-x_{2}=3-1-0=2,
$$

meaning that if $(1,0,3)$ is part of the final selection, then the coalition $\{1,2\}$ is allocated in total 2 less than its worth. Table 1 shows the excesses for all coalitions in $F$ w.r.t. the two leading allocations.

\begin{tabular}{c|ccc}
$x$ & $E(\{1,2\}, x)$ & $E(\{1,3\}, x)$ & $E(\{2,3\}, x)$ \\
\hline$(1,0,3)$ & 2 & 0 & 2 \\
$(0,2,3)$ & 1 & 1 & 0
\end{tabular}

Table 1: The excesses of all feasible coalitions in Example 1 with respect to the two leading allocations.

In order to determine $\mathscr{N}^{F}$, we consider the sorted excess vectors $\theta(F,(1,0,3))=(2,2,0)$ and $\theta(F,(0,2,3))=(1,1,0)$. The lexicographical minimum is $(1,1,0)$, which means that $(0,2,3)$ is the unique element of $\mathscr{N}^{F}$. In order to determine $\mathscr{N}^{L}$, we consider the sorted excess vectors $\theta(L,(1,0,3))=(2,0)$ and $\theta(L,(0,2,3))=(1,0)$ that contains only the excesses of leading coalitions. The lexicographical minimum is $(1,0)$, which means that $(0,2,3)$ is also the unique element of $\mathscr{N}^{L}$. Thus

$$
\mathscr{N}^{F}=\mathscr{N}^{L}=\{(\{2,3\},(0,2,3))\} .
$$


In the example above, leading selections exist. An important question is whether leading selections always exist. The following example illustrates that this is not guaranteed in general.

Example 2 Consider a cooperative selection situation $(N, F, V)$ with $N=\{1, \ldots, 3\}, F=$ $\{\{1,2\},\{1,3\},\{2,3\}\}, v(\{i\})=\frac{1}{2}$ for all $i \in N$ and

$$
V(S)= \begin{cases}\{(2,1,0)\} & \text { if } S=\{1,2\} \\ \{(0,2,1)\} & \text { if } S=\{2,3\} \\ \{(1,0,2)\} & \text { if } S=\{1,3\}\end{cases}
$$

The selections are $\left(\{1,2\},\left(2,1, \frac{1}{2}\right)\right),\left(\{2,3\},\left(\frac{1}{2}, 2,1\right)\right)$ and $\left(\{1,3\},\left(1, \frac{1}{2}, 2\right)\right)$, and, as Figure 2 illustrates, each of these selections is blocked by another one. In particular, the blocking graph contains no sinks and so there are no leading selections.

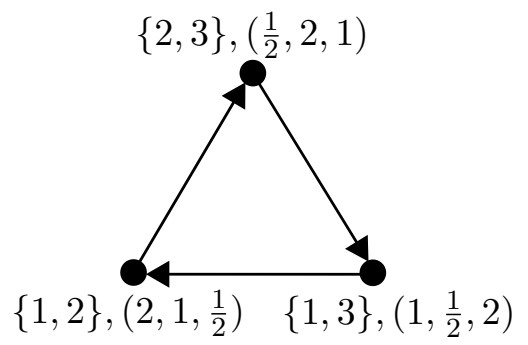

Figure 2: Blocking graph a cooperative selection situation that has no leading coalitions.

The existence of at least one sink in the blocking graph is guaranteed as long as the graph does not contain any cycles. Thus leading selections always exists for cooperative selection situations with acyclic blocking graphs. A practical example of this is vertical collaboration in a multi-tier supply chain with one agent per tier, where only coalitions containing agents of adjacent tiers are allowed (i.e. direct supplier-buyer relationships are required to form coalitions). In this case the blocking graph will contain no cycles (and thus leading coalitions will exist), since the existence of a cycle will necessarily imply a coalition consisting of agents from non-adjacent tiers - e.g. a manufacturer's supplier and one of the manufacturer's customers form a coalition without the manufacturer, which would not be allowed.

The following example illustrates the difference between the finite case and the polytope case, showing in particular that if the finite payoff sets from Example 1 are replaced by their respective convex hulls, the set of leading selections, and therefore also the nucleolus selections, can be different.

Example 3 Consider the cooperative selection situation $(N, F, V)$ with $N=\{1,2,3\}, F=$ $\{\{1,2\},\{1,3\},\{2,3\}\}, v(\{i\})=0$ for all $i \in N$, and

$$
V(S)= \begin{cases}\operatorname{conv}(\{(0,3,0),(1,2,0)\}) & \text { if } S=\{1,2\}, \\ \operatorname{conv}(\{(0,0,4),(1,0,3)\}) & \text { if } S=\{1,3\}, \\ \operatorname{conv}(\{(0,2,3),(0,4,1)\}) & \text { if } S=\{2,3\} .\end{cases}
$$

In this case the payoff sets are not finite, and it is not possible to construct a blocking graph. However, the following observations can be made in order to identify the leading selections:

- Any selection $(\{1,2\}, x)$ is blocked by the selection $(\{2,3\},(0,4,1))$, and thus $\{1,2\}$ can never be a leading coalition.

- Any selection $(\{1,3\}, x)$ with $x_{1}<1$ is blocked by the selection $(\{1,2\},(1,2,0))$. The selection $(\{1,3\},(1,0,3))$ is not blocked by another selection. 
- The selection $(\{2,3\}, x)$ with $x_{3}<3$ is blocked by $(\{1,3\},(1,0,3))$ and the selection $(\{2,3\},(0,2,3))$ is blocked by the selection $\left(\{1,2\},\left(\frac{1}{2}, 2 \frac{1}{2}, 0\right)\right)$.

Thus $\mathscr{L}=\{(\{1,3\},(1,0,3))\}$.

For the finite case, the blocking graph provides an algorithmic means of identifying the leading selections. We want to remark that for the polytope case, leading coalitions can be identified using linear programming as follows. In case $V(S)$ is a convex polytope, verifying whether or not coalition $S$ is leading boils down to checking if the optimal value of the linear program below is at most equal to 0 , for each $T \in F \backslash\{S\}$, with $T \cap S \neq \varnothing$.

$\begin{array}{lll}\max & \epsilon & \\ \text { subject to } & & i \in T \cap S \\ y_{i} & \geq \max _{x \in V(S)} x_{i}+\epsilon & i \in T \backslash S \\ y_{i} \geq v(\{i\})+\epsilon & \\ y & \in V(T) & \end{array}$

\section{Application to linear production processes}

In this section we apply the methodology of Section 2 to so-called linear production processes where agents can collaborate in producing and selling goods by pooling their production resources. Consider a set of products $P$ that can be produced and sold at fixed market prices, where $c \in \mathbb{R}_{+}^{P}$ is a vector specifying the fixed market price $c_{p}$ of product $p \in P$. For production, a set of resources $R$ is needed, where $A \in \mathbb{R}_{+}^{R \times P}$ is a linear production matrix specifying the amount $A_{r p}$ of resource $r \in R$ needed to produce one unit of product $p \in P$. The assumption is further made that for each product $p \in P$ there exists at least one resource $r \in R$ such that $A_{r p}>0$. Given a resource bundle $b \in \mathbb{R}_{+}^{R}$, i.e. a vector in which $b_{r}$ specifies the amount of resource $r \in R$ that is available, the maximum revenue that can be generated by the process is given by the optimal value $\operatorname{LP}^{*}(A, b, c)$ to the linear maximization program

$$
\max c x \quad \text { s.t. } A x \leq b, \quad x \geq 0
$$

where $x \in \mathbb{R}_{+}^{P}$ represents a production plan, and $x_{p}$ represents the amount of product $p$ produced. Using duality, $\operatorname{LP}^{*}(A, b, c)$ also equals the optimal value of the linear minimization problem

$$
\min b y \quad \text { s.t. } \quad y^{\top} A \geq c, \quad y \geq 0
$$

with $y \in \mathbb{R}_{+}^{R}$ representing the shadow prices. As we shall see, the dual solutions to this linear program will be useful, and we will denote by $Y(A, b, c)$ all the dual optimal solutions.

Finally, we consider $N$ to be a set of agents. Each agent owns a certain amount of each resource, where $b^{i} \in \mathbb{R}_{+}^{R}$ is a resource bundle specifying the amount $b_{r}^{i}$ of resource $r \in R$ that agent $i \in N$ owns. We assume that $\sum_{i \in N} b_{r}^{i}>0$ for each resource $r \in R$, i.e. a nonzero amount of each resource is owned by at least one agent.

The tuple $\left(N, R, P, A,\left\{b^{i}\right\}_{i \in N}, c\right)$ is called a linear production process and it is associated with a linear production game, which is a cooperative game $(N, w)$ where

$$
w(S)=\mathrm{LP}^{*}\left(A, \sum_{i \in S} b^{i}, c\right),
$$

for all $S \subseteq N$. In other words, it represents horizontal collaboration where the agents can form coalitions in order to pool resources. Collaborative gains are achieved through more efficient utilization of resources, allowing coalitions to potentially produce and sell more than the sum of the outputs if the agents acted in isolation. The question then remains as to how to share the 
gains of collaboration in a fair way. Fortunately, an attractive, context specific allocation rule for linear production games has been developed, namely the Owen set, due to Owen (1975). For a detailed analysis of the attractive properties of the Owen set we refer to Van Gellekom et al. (2000). The Owen set of a linear production process is given by

$$
\operatorname{Owen}\left(N, R, P, A,\left\{b^{i}\right\}_{i \in N}, c\right)=\left\{\left\{y b^{i}\right\}_{i \in N} \mid y \in Y\left(A, \sum_{i \in N} b^{i}, c\right)\right\},
$$

i.e. each agents receives a payoff equal to the shadow value of its own resource bundle when using optimal shadow prices w.r.t. (2) as benchmarks. The Owen set has an appealing stability property, namely that it is a subset of the core of the linear production game $(N, w)$. That is, any $x \in \operatorname{Owen}\left(N, R, P, A,\left\{b^{i}\right\}_{i \in N}, c\right)$ satisfies

$$
\sum_{i \in N} x_{i}=w(N) \text { and } \sum_{i \in S} x_{i} \geq w(S)
$$

for all $S \subseteq N$.

Given a linear production process $\left(N, R, P, A,\left\{b^{i}\right\}_{i \in N}, c\right)$, we consider a corresponding cooperative selection situation $(N, F, V)$ with $F \subseteq 2^{N}$ such that $|S| \geq 2$ for all $S \in F$ and

$$
V(S)=\operatorname{Owen}\left(S, R, P, A,\left\{b^{i}\right\}_{i \in S}, c\right)
$$

for each $S \in F$ and $S=\{i\}, i \in N$. In other words, a linear production game is associated to each feasible leading coalition and the Owen set is used as the means of distributing the collaborative gains.

\subsection{Examples}

We next present some examples to illustrate the various outcomes that may be observed when applying the methodology of Section 2 to linear production processes. We will pay special attention to the overall profit generated by a selection $(S, x)$, given by

$$
v^{*}(S)=v(S)+\sum_{i \notin S} v(\{i\}) .
$$

Example 4 in particular shows that the selection that maximizes overall profit can be blocked, and, as a consequence, can not be a leading selection nor a nucleolus selection.

Example 4 Consider a linear production process with 3 products, 7 resources, where the production matrix $A$ is given by

$$
A=\left[\begin{array}{ccc}
7 & 0 & 5 \\
2 & 0 & 2 \\
2 & 0 & 6 \\
9 & 10 & 9 \\
0 & 0 & 7 \\
9 & 0 & 6 \\
4 & 0 & 8
\end{array}\right]
$$

and where the market prices are given by $c^{\top}=\left[\begin{array}{ll}8 & 7\end{array}\right]$. Moreover, let $N=\{1, \ldots, 6\}$ and let the resources bundles the agents own be given by

$$
b^{1}=\left[\begin{array}{l}
0 \\
8 \\
9 \\
4 \\
0 \\
8 \\
0
\end{array}\right] \quad b^{2}=\left[\begin{array}{l}
5 \\
3 \\
0 \\
9 \\
4 \\
5 \\
8
\end{array}\right] \quad b^{3}=\left[\begin{array}{l}
0 \\
4 \\
5 \\
7 \\
0 \\
5 \\
8
\end{array}\right] \quad b^{4}=\left[\begin{array}{c}
10 \\
0 \\
0 \\
3 \\
0 \\
9 \\
0
\end{array}\right] \quad b^{5}=\left[\begin{array}{c}
2 \\
7 \\
1 \\
8 \\
0 \\
10 \\
0
\end{array}\right], \text { and } b^{6}=\left[\begin{array}{l}
6 \\
0 \\
5 \\
7 \\
9 \\
1 \\
0
\end{array}\right]
$$


We assume there are 7 feasible leading coalitions each consisting of two agents. These coalitions are shown in the first column of Table 2. Solving the respective linear programs and using Owen vectors, we find that the associated cooperative selection situation is as shown in Table 2. In this example the Owen set consists of a unique element for all feasible leading coalitions.

\begin{tabular}{ccc|l|lcccccc}
$S$ & $v(S)$ & $v^{*}(S)$ & \multicolumn{10}{c}{$V(S)$} \\
\hline 2,3$\}$ & 12.41 & 27.81 & $\{($ & 0 & 7.51 & 4.9 & 0 & 0 & 0 & )$\}$ \\
$\{1,5\}$ & 8.4 & 26.6 & $\{($ & 2.8 & 0 & 0 & 0 & 5.6 & 0 & )$\}$ \\
$\{3,5\}$ & 10.99 & 27.09 & $\{($ & 0 & 0 & 4.9 & 0 & 6.09 & 0 & )$\}$ \\
$\{4,5\}$ & 7.7 & 26.6 & $\{($ & 0 & 0 & 0 & 2.1 & 5.6 & 0 & )$\}$ \\
$\{2,6\}$ & 12.33 & 27.73 & $\{($ & 0 & 7.24 & 0 & 0 & 0 & 5.09 & )$\}$ \\
$\{3,6\}$ & 10.93 & 27.73 & $\{($ & 0 & 0 & 5.84 & 0 & 0 & 5.09 & )$\}$ \\
$\{4,6\}$ & 7 & 26.6 & $\{($ & 0 & 0 & 0 & 2.1 & 0 & 4.9 & )$\}$
\end{tabular}

\begin{tabular}{c|c}
$i$ & $v(\{i\})$ \\
\hline 0 & 2.8 \\
1 & 6.3 \\
2 & 4.9 \\
3 & 2.1 \\
4 & 5.6 \\
5 & 4.9
\end{tabular}

Table 2: The cooperative selection situation in Example 4.

Using a blocking graph it can be seen that the set of leading selections is given by

$$
\begin{aligned}
\mathscr{L}=\{ & (\{1,5\},(2.8,6.3,4.9,2.1,5.6,4.9)) \\
& (\{4,5\},(2.8,6.3,4.9,2.1,5.6,4.9)) \\
& (\{2,6\},(2.8,7.24,4.9,2.1,5.6,5.09)) \\
& (\{3,6\},(2.8,6.3,5.84,2.1,5.6,5.09))\} .
\end{aligned}
$$

Note that the selection that maximizes overall profit, namely $(\{2,3\},(2.8,7.51,4.9,2.1,5.6,4.9))$, is not leading. Next, the excesses of all feasible leading coalitions with respect to the four leading allocations are given in Table 3, together with the corresponding ordered vector of excesses. From this it follows that

$$
\mathscr{N}^{F}=\{(\{3,6\},(2.8,6.3,5.84,2.1,5.6,5.09))\}
$$

and

$$
\begin{aligned}
\mathscr{N}^{L}=\{( & (\{2,6\},(2.8,7.24,4.9,2.1,5.6,5.09)), \\
& (\{3,6\},(2.8,6.3,5.84,2.1,5.6,5.09))\} .
\end{aligned}
$$

\begin{tabular}{|c|c|c|c|c|c|c|c|c|c|c|c|c|}
\hline$x_{1}$ & $x_{2}$ & $x_{3}$ & $x_{4}$ & $x_{5}$ & $x_{6}$ & \multicolumn{7}{|c|}{ Excesses sorted (excesses of coalitions in $F \backslash L$ in brackets) } \\
\hline 2.8 & 6.3 & 4.9 & 2.1 & 5.6 & 4.9 & $(1.21)$ & 1.13 & 1.13 & $(0.49)$ & 0 & 0 & $(0)$ \\
\hline 2.8 & 6.3 & 4.9 & 2.1 & 5.6 & 4.9 & $(1.21)$ & 1.13 & 1.13 & $(0.49)$ & 0 & 0 & $(0)$ \\
\hline 2.8 & 7.24 & 4.9 & 2.1 & 5.6 & 5.09 & 0.94 & $(0.49)$ & $(0.27)$ & 0 & 0 & 0 & $(-0.19)$ \\
\hline 2.8 & 6.3 & 5.84 & 2.1 & 5.6 & 5.09 & 0.94 & $(0.27)$ & 0 & 0 & 0 & $(-0.19)$ & $(-0.46)$ \\
\hline
\end{tabular}

Note that $\mathscr{N}^{F}$ is a strict subset of $\mathscr{N}^{L}$.

Leading allocations

Feasible leading coalitions

\begin{tabular}{cccccc|ccccccc}
$x_{1}$ & $x_{2}$ & $x_{3}$ & $x_{4}$ & $x_{5}$ & $x_{6}$ & $\{1,5\}$ & $\{4,5\}$ & $\{2,6\}$ & $\{3,6\}$ & $\{2,3\}$ & $\{3,5\}$ & $\{4,6\}$ \\
\hline 2.8 & 6.3 & 4.9 & 2.1 & 5.6 & 4.9 & 0 & 0 & 1.13 & 1.13 & 1.21 & 0.49 & 0 \\
2.8 & 6.3 & 4.9 & 2.1 & 5.6 & 4.9 & 0 & 0 & 1.13 & 1.13 & 1.21 & 0.49 & 0 \\
2.8 & 7.24 & 4.9 & 2.1 & 5.6 & 5.09 & 0 & 0 & 0 & 0.94 & 0.27 & 0.49 & -0.19 \\
2.8 & 6.3 & 5.84 & 2.1 & 5.6 & 5.09 & 0 & 0 & 0.94 & 0 & 0.27 & -0.46 & -0.19
\end{tabular}

Table 3: The excesses corresponding to the four leading allocations in Example 4.

Example 4 exhibits the feature that $\mathscr{N}^{F} \subset \mathscr{N}^{L}$. In the computational results later on we show that this is the case almost all of the time. However, the following example shows that this nesting property does not hold in general. In fact, the following example shows it is possible that $\mathscr{N}^{F} \cap \mathscr{N}^{L}=\varnothing$. 
Example 5 Consider a linear production process with 8 products, 4 resources, where the production matrix $A$ is given by

$$
A=\left[\begin{array}{cccccccc}
5 & 0 & 3 & 3 & 1 & 0 & 9 & 0 \\
0 & 0 & 7 & 10 & 0 & 10 & 0 & 3 \\
0 & 0 & 6 & 6 & 3 & 5 & 1 & 0 \\
10 & 10 & 0 & 0 & 0 & 0 & 5 & 8
\end{array}\right]
$$

and where the market prices are given by $c^{\top}=\left[\begin{array}{lllllll}10 & 8 & 7 & 1 & 4 & 6 & 3\end{array}\right]$. Moreover, there are 4 agents and the individual resource bundles they own are given by

$$
b^{1}=\left[\begin{array}{l}
8 \\
9 \\
1 \\
3
\end{array}\right] \quad b^{2}=\left[\begin{array}{l}
4 \\
4 \\
5 \\
5
\end{array}\right] \quad b^{3}=\left[\begin{array}{l}
0 \\
8 \\
5 \\
0
\end{array}\right], \text { and } b^{4}=\left[\begin{array}{l}
0 \\
7 \\
8 \\
1
\end{array}\right]
$$

The associated cooperative selection situation is provided in Table 4 and the excesses of all leading allocations are shown in Table 5. We can see here that

$$
\mathscr{N}^{F}=\{(\{2,3,4\},(4.33,11.98,6,10.47))\}
$$

\begin{tabular}{|c|c|c|c|c|c|c|c|c|c|}
\hline$S$ & $v(S)$ & $v^{*}(S)$ & \multicolumn{5}{|c|}{$V(S)$} & $i$ & $v(\{i\})$ \\
\hline$\{1,2\}$ & 16 & 25.8 & 4.33 & 11.67 & 0 & 0 & )\} & 0 & 4.33 \\
\hline$\{1,2,3\}$ & 22.67 & 27.67 & 4.33 & 11.67 & 6.67 & 0 & )\} & 1 & 11.62 \\
\hline$\{2,4\}$ & 22.45 & 31.58 & $\{($ & 11.98 & 0 & 10.47 & )\} & 2 & 4.8 \\
\hline$\{1,2,4\}$ & 27.67 & 32.47 & 4.33 & 11.67 & 0 & 11.67 & )$\}$ & 3 & 5 \\
\hline$\{2,3,4\}$ & 28.45 & 32.78 & $\{($ & 11.98 & 6 & 10.47 & )$\}$ & & \\
\hline
\end{tabular}

and

$$
\mathscr{N}^{L}=\{(\{1,2,4\},(4.33,11.67,4.8,11.67))\}
$$

\begin{tabular}{|c|c|c|c|c|c|c|c|c|c|}
\hline & \multicolumn{4}{|c|}{ Leading allocations } & \multicolumn{5}{|c|}{ Feasible leading coalitions } \\
\hline & $x_{1}$ & $x_{2}$ & $x_{3}$ & $x_{4}$ & $\{2,4\}$ & $\{1,2,4\}$ & $\{2,3,4\}$ & $\{1,2\}$ & $\{1,2,3\}$ \\
\hline & 4.33 & 11.98 & 4.8 & 10.47 & 0 & 0.88 & 1.2 & -0.31 & 1.56 \\
\hline & 4.33 & 11.67 & 4.8 & 11.67 & -0.88 & 0 & 0.32 & 0 & 1.87 \\
\hline & 4.33 & 11.98 & 6 & 10.47 & 0 & 0.88 & 0 & -0.31 & 0.36 \\
\hline$x_{1}$ & $x_{2}$ & $x_{3}$ & $x_{4}$ & Exce & ses sorte & d (excess & s of coali & ons in $F$ & $L$ in brackets) \\
\hline 4.33 & 11.98 & 4.8 & 10.47 & & $(1.56)$ & 1.2 & $\begin{array}{cc}0.88 \\
\end{array}$ & $(-0.31)$ & \\
\hline 4.33 & 11.67 & 4.8 & 11.67 & & $(1.87)$ & 0.32 & (0) & -0.88 & \\
\hline 4.33 & 11.98 & 6 & 10.47 & & 0.88 & $(0.36)$ & 0 & $(-0.31)$ & \\
\hline
\end{tabular}

Table 4: The cooperative selection situation in Example 5.

Table 5: The excesses corresponding to the leading selections in Example 5.

\section{Computational results}

In order to gain more insight into leading selections and nucleolus selections for linear production processes, we apply our methodology on a set of randomly generated instances and summarize the results in this section. In particular we are interested in answering the following questions. 
How likely is it that there exists no leading selection? How likely is it that there exists only one leading selection? How likely is it that there is exactly one nucleolus selection? Finally, we also investigate how often $\mathscr{N}^{F}$ is a subset of $\mathscr{N}^{L}$, as we observed in Example 4.

For each $|N| \in\{4,5,6,7\}$ we generate 1000 linear production process instances in the following way. The number of products and the number of resources are drawn from $U(3,8)$. Each element of $A$ and $b^{i}$ is drawn from $U(1,10)$ with probability 0.7 ; otherwise it is zero. The inclusion of zeroes in these matrices is done to reflect the fact that typically not all resources are required for each product, and that not all agents necessarily own a nonzero amount of every possible resource. We repeat the generation of $A$ if either $\sum_{p \in P} A_{r p}=0$ for some $r \in R$ or $\sum_{r \in R} A_{r p}=0$ for some $p \in P$ in order to ensure that all resources are used and that each product requires at least one resource. Moreover, we repeat the generation of $b^{i}$ if $\sum_{r \in R} b_{r}^{i} A_{r p}=0$ for all $p \in P$ in order to ensure that each agent can produce at least one product on its own. Finally, each element of $c$ is drawn from $U(1,10)$.

For generating $F$ we consider two types of restrictions, namely size restrictions, which puts an upper bound on the size of a coalition in order to curb cooperation complexity, and relational restrictions, which makes certain combinations of agents infeasible due to issues such as trust and information sensitivity. We consider three classes of size restrictions, namely one where only pairs of agents can collaborate, one where only triplets of agents can collaborate, and one where only pairs and triplets can collaborate. We consider two classes of relational restrictions, namely one where there is none, so all coalitions within size restrictions are feasible, and one where only half of the coalitions within the size restrictions are feasible, this half being randomly selected in each case. We use the notation $\rho$ to denote the fraction of coalitions within in the size restrictions that are feasible, and the two considered cases are therefore given by $\rho=1$ and $\rho=0.5$, respectively. Table 6 shows the different sizes for $F$ that results from these classes of restrictions.

\begin{tabular}{cc|cc} 
& & \multicolumn{2}{|c}{$|F|$} \\
$|N|$ & Coalition size & $\rho=1$ & $\rho=0.5$ \\
\hline 4 & 2 & 6 & 3 \\
4 & 3 & 4 & 2 \\
4 & 2 or 3 & 10 & 5 \\
5 & 2 & 10 & 5 \\
5 & 3 & 10 & 5 \\
5 & 2 or 3 & 20 & 10 \\
6 & 2 & 15 & 7 \\
6 & 3 & 20 & 10 \\
6 & 2 or 3 & 35 & 17 \\
7 & 2 & 21 & 10 \\
7 & 3 & 35 & 17 \\
7 & 2 or 3 & 56 & 28
\end{tabular}

Table 6: Details on the classes of instances considered.

Each of the 4000 linear production process instances is then coupled with each of the six cases of restrictions (three size restrictions and two relational restrictions) to create 24000 cooperative selection situation instances. For each instance, $V(S)$ is calculated for each $S \in F$ by solving for the corresponding Owen set. For solving the underlying linear programs, CPLEX 12.10 was used. In order to obtain all dual solutions (as necessary for computing the Owen set), all possible sets of basic variables in the dual were simply enumerated and for each it was checked whether it was optimal in order to obtain the vertices of the optimal face of the dual linear program. Even though there exists more sophisticated and efficient methods for finding all optimal solutions to a linear program (see e.g. Lee et al. (2000)), this simple approach is sufficient in our case since the linear programs we consider are relatively small. Finally, we restricted the study to those instances where $V(S)$ contains a single point for all $S \in F$. This has led to 18914 instances out 
of the original set of $24000(79 \%)$.

Table 7 shows results on the number of leading selections for the different classes of instances, namely the percentage of instances for which at least one leading selection exists $(|\mathscr{L}|>0)$, the average percentage of feasible coalitions that are leading $(|L| /|F|)$, and the percentage of instance where there is only one leading selection $(|\mathscr{L}|=1)$. The table shows that in most instances there exists at least one leading coalition, and that the proposed methodology will provide at least one nucleolus selection. However, as the number of agents increases, the percentage of instances with no leading coalitions tends to increase. The same can be observed if the coalition sizes increase and if $\rho$ increases. The table further shows that on average the majority of feasible coalitions are leading, and that it is very unlikely that there exists a single leading selection. This justifies the need for the second step in our methodology, where nucleolus selections are calculated.

\begin{tabular}{|c|c|c|c|c|c|c|c|}
\hline \multirow[b]{2}{*}{ Coalition size } & \multirow[b]{2}{*}{$|N|$} & \multicolumn{2}{|c|}{$|\mathscr{L}|>0$} & \multicolumn{2}{|c|}{$|L| /|F|$} & \multicolumn{2}{|c|}{$|\mathscr{L}|=1$} \\
\hline & & $\rho=0.5$ & $\rho=1$ & $\rho=0.5$ & $\rho=1$ & $\rho=0.5$ & $\rho=1$ \\
\hline \multirow{5}{*}{2} & 4 & $100 \%$ & $97 \%$ & $77 \%$ & $61 \%$ & $21 \%$ & $19 \%$ \\
\hline & 5 & $99 \%$ & $95 \%$ & $68 \%$ & $52 \%$ & $11 \%$ & $15 \%$ \\
\hline & 6 & $100 \%$ & $96 \%$ & $64 \%$ & $51 \%$ & $8 \%$ & $11 \%$ \\
\hline & 7 & $99 \%$ & $96 \%$ & $63 \%$ & $53 \%$ & $6 \%$ & $11 \%$ \\
\hline & age & $99 \%$ & $96 \%$ & $68 \%$ & $54 \%$ & $12 \%$ & $14 \%$ \\
\hline \multirow{5}{*}{3} & 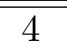 & $100 \%$ & $100 \%$ & $\overline{95 \%}$ & $88 \%$ & $10 \%$ & $0 \%$ \\
\hline & 5 & $99 \%$ & $98 \%$ & $76 \%$ & $65 \%$ & $7 \%$ & $4 \%$ \\
\hline & 6 & $98 \%$ & $97 \%$ & $64 \%$ & $56 \%$ & $7 \%$ & $8 \%$ \\
\hline & 7 & $94 \%$ & $89 \%$ & $57 \%$ & $53 \%$ & $8 \%$ & $7 \%$ \\
\hline & age & $98 \%$ & $96 \%$ & $73 \%$ & $66 \%$ & $8 \%$ & $5 \%$ \\
\hline \multirow{5}{*}{2 or 3} & 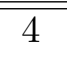 & $100 \%$ & $100 \%$ & $74 \%$ & $64 \%$ & $8 \%$ & $6 \%$ \\
\hline & 5 & $98 \%$ & $96 \%$ & $60 \%$ & $52 \%$ & $6 \%$ & $7 \%$ \\
\hline & 6 & $96 \%$ & $95 \%$ & $54 \%$ & $51 \%$ & $8 \%$ & $7 \%$ \\
\hline & 7 & $94 \%$ & $91 \%$ & $54 \%$ & $55 \%$ & $8 \%$ & $5 \%$ \\
\hline & age & $97 \%$ & $95 \%$ & $60 \%$ & $55 \%$ & $8 \%$ & $6 \%$ \\
\hline
\end{tabular}

Table 7: Results on leading selections for different classes of instances.

Table 8 shows results on the number of the nucleolus selections in $\mathscr{N}^{F}$ and $\mathscr{N}^{L}$ for all instances for which at least one leading selection exists. It shows the percentage of those instances for which $\mathscr{N}^{F}$ or $\mathscr{N}^{L}$ consists of a single selection, as well as the percentage of instances where $\mathscr{N}^{F}=\mathscr{N}^{L}$. It can be seen that in the large majority of instances both $\mathscr{N}^{F}$ and $\mathscr{N}^{L}$ consist of a single selection. It is thus relatively likely that the proposed methodology will result in a unique answer for linear production processes. The table also shows that the two nucleoli are almost always equal.

From Table 8 it can also be seen that for larger coalition sizes and larger $\rho$ the likelyhood of $\left|\mathscr{N}^{F}\right|=1$ and $\left|\mathscr{N}^{L}\right|=1$ decreases. In other words, with fewer restrictions it is less likely to obtain a unique selection. Thus, our methodology seems better suited for cases where there are more restrictions.

Finally, we study the nucleolus selections in relation to overall profit generated. We define

$$
\Pi=\{S \in F \mid v(S) \geq v(T), \forall T \in F\}
$$

as the set of feasible leading coalitions generating the highest profit for their members, while we define

$$
\Pi^{*}=\left\{S \in F \mid v^{*}(S) \geq v^{*}(T), \forall T \in F\right\}
$$

as the set of coalitions that would, if part of a selection, generate the highest profit for all agents. We dinstinguish between four cases: 


\begin{tabular}{|c|c|c|c|c|c|c|c|}
\hline \multirow[b]{2}{*}{ Coalition size } & \multirow[b]{2}{*}{$|N|$} & \multicolumn{2}{|c|}{$\left|\mathscr{N}^{F}\right|=1$} & \multicolumn{2}{|c|}{$\left|\mathscr{N}^{L}\right|=1$} & \multicolumn{2}{|c|}{$\mathscr{N}^{F}=\mathscr{N}^{L}$} \\
\hline & & $\rho=0.5$ & $\rho=1$ & $\rho=0.5$ & $\rho=1$ & $\rho=0.5$ & $\rho=1$ \\
\hline \multirow{5}{*}{2} & 4 & $84 \%$ & $75 \%$ & $84 \%$ & $75 \%$ & $99 \%$ & $97 \%$ \\
\hline & 5 & $83 \%$ & $76 \%$ & $83 \%$ & $76 \%$ & $97 \%$ & $96 \%$ \\
\hline & 6 & $83 \%$ & $73 \%$ & $82 \%$ & $72 \%$ & $96 \%$ & $93 \%$ \\
\hline & 7 & $79 \%$ & $68 \%$ & $79 \%$ & $67 \%$ & $95 \%$ & $93 \%$ \\
\hline & age & $82 \%$ & $73 \%$ & $82 \%$ & $73 \%$ & $97 \%$ & $95 \%$ \\
\hline \multirow{5}{*}{3} & $\overline{4}$ & $85 \%$ & $70 \%$ & $85 \%$ & $70 \%$ & $100 \%$ & $98 \%$ \\
\hline & 5 & $78 \%$ & $71 \%$ & $78 \%$ & $71 \%$ & $95 \%$ & $94 \%$ \\
\hline & 6 & $76 \%$ & $69 \%$ & $76 \%$ & $69 \%$ & $91 \%$ & $90 \%$ \\
\hline & 7 & $72 \%$ & $64 \%$ & $72 \%$ & $63 \%$ & $90 \%$ & $91 \%$ \\
\hline & age & $78 \%$ & $69 \%$ & $78 \%$ & $68 \%$ & $94 \%$ & $93 \%$ \\
\hline \multirow{5}{*}{2 or 3} & 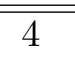 & 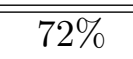 & 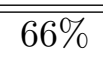 & $\overline{72 \%}$ & $67 \%$ & $\overline{988 \%}$ & $96 \%$ \\
\hline & 5 & $76 \%$ & $68 \%$ & $76 \%$ & $68 \%$ & $95 \%$ & $95 \%$ \\
\hline & 6 & $73 \%$ & $63 \%$ & $73 \%$ & $63 \%$ & $92 \%$ & $92 \%$ \\
\hline & 7 & $68 \%$ & $57 \%$ & $67 \%$ & $57 \%$ & $91 \%$ & $92 \%$ \\
\hline & age & $72 \%$ & $64 \%$ & $72 \%$ & $64 \%$ & $94 \%$ & $94 \%$ \\
\hline
\end{tabular}

Table 8: Results on the nucleolus selections for different classes of instances.

- Case 1: There is a selection $(S, x) \in \mathscr{N}^{F}$ such that $S \in \Pi$.

- Case 2: There is a selection $(S, x) \in \mathscr{N}^{\mathscr{L}}$ such that $S \in \Pi$.

- Case 3: There is a selection $(S, x) \in \mathscr{N}^{F}$ such that $S \in \Pi^{*}$.

- Case 4: There is a selection $(S, x) \in \mathscr{N}^{\mathscr{L}}$ such that $S \in \Pi^{*}$.

Table 9 shows the percentage of instances where each of the four cases is observed. What the table shows is that it is often the case that a coalition in $\Pi^{*}$ is a nucleolus selection, while it is considerably less likely that the more "myopic" selections containing a coalition in $\Pi$ are nucleolus selections.

Case 1

Case 2

Case 3

Case 4

\begin{tabular}{|c|c|c|c|c|c|c|c|c|c|}
\hline Coalition siz & $|N|$ & $\rho=0.5$ & $\rho=1$ & $\rho=0.5$ & $\rho=1$ & $\rho=0.5$ & $\rho=1$ & $\rho=0.5$ & $\rho=1$ \\
\hline \multirow{5}{*}{2} & 4 & $59 \%$ & $40 \%$ & $58 \%$ & $41 \%$ & $90 \%$ & $81 \%$ & $90 \%$ & $81 \%$ \\
\hline & 5 & $48 \%$ & $35 \%$ & $48 \%$ & $35 \%$ & $86 \%$ & $74 \%$ & $87 \%$ & $74 \%$ \\
\hline & 6 & $38 \%$ & $28 \%$ & $39 \%$ & $28 \%$ & $86 \%$ & $70 \%$ & $86 \%$ & $72 \%$ \\
\hline & 7 & $32 \%$ & $26 \%$ & $32 \%$ & $26 \%$ & $79 \%$ & $70 \%$ & $80 \%$ & $70 \%$ \\
\hline & Average & $44 \%$ & $32 \%$ & $44 \%$ & $32 \%$ & $85 \%$ & $74 \%$ & $86 \%$ & $74 \%$ \\
\hline \multirow{5}{*}{3} & 4 & $70 \%$ & $55 \%$ & $70 \%$ & $54 \%$ & $96 \%$ & $90 \%$ & $96 \%$ & $90 \%$ \\
\hline & 5 & $45 \%$ & $36 \%$ & $45 \%$ & $37 \%$ & $75 \%$ & $74 \%$ & $76 \%$ & $73 \%$ \\
\hline & 6 & $29 \%$ & $26 \%$ & $30 \%$ & $26 \%$ & $66 \%$ & $66 \%$ & $66 \%$ & $65 \%$ \\
\hline & 7 & $26 \%$ & $22 \%$ & $26 \%$ & $22 \%$ & $62 \%$ & $61 \%$ & $62 \%$ & $59 \%$ \\
\hline & Average & $43 \%$ & $35 \%$ & $43 \%$ & $35 \%$ & $75 \%$ & $73 \%$ & $75 \%$ & $72 \%$ \\
\hline \multirow{5}{*}{2 or 3} & 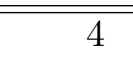 & $\overline{62 \%}$ & $\bar{~} \overline{53 \%}$ & 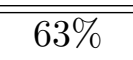 & $54 \%$ & 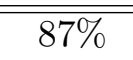 & $87 \%$ & $\begin{array}{c}88 \% \\
\end{array}$ & $89 \%$ \\
\hline & 5 & $42 \%$ & $36 \%$ & $43 \%$ & $36 \%$ & $72 \%$ & $71 \%$ & $73 \%$ & $71 \%$ \\
\hline & 6 & $29 \%$ & $29 \%$ & $30 \%$ & $29 \%$ & $64 \%$ & $68 \%$ & $65 \%$ & $67 \%$ \\
\hline & 7 & $25 \%$ & $28 \%$ & $24 \%$ & $28 \%$ & $60 \%$ & $65 \%$ & $59 \%$ & $65 \%$ \\
\hline & Average & $40 \%$ & $36 \%$ & $40 \%$ & $37 \%$ & $71 \%$ & $73 \%$ & $71 \%$ & $73 \%$ \\
\hline
\end{tabular}

Table 9: The percentage of instances where nucleolus selections consist of coalitions that generate the highest profit. 
The computational study shows that our methodology will in many cases successfully identify a final leading selection, also if there exists many leading selections. Furthermore, in a large number of instances the nucleolus selections consist of a leading selection that maximizes overall profit among all agents. Thus in many cases our approach leads to overall efficiency. This provides some indication that the proposed methodology is especially well suited to situations where the set of feasible coalitions is relatively small, i.e. where there are many restrictions to collaboration (on size and relational aspects). As discussed in the introduction, this is ubiquitous in supply chains.

\section{Conclusion}

In this paper we present a new model, namely a cooperative selection situation, in order to address a specific problem in supply chain collaboration of which among a restricted set of coalitions should form. The focus is on describing a new methodology based on the concept of a leading selection which consists of a leading coalition together with a leading allocation. A leading selection has the property that it can not be blocked by another leading selection. We show how leading selections can be identified algorithmically, and we propose a nucleolusbased procedure for narrowing the set of leading selections down to set of final selections, called nucleolus selections. We apply the methodology to linear production processes and present results through the use of some examples and a computational study based on a large set of randomly generated instances. Our results show that the methodology is able to narrow the set of potentially leading selections down to a single leading selection in most cases, and that quite often the leading selection that maximizes the overall supply chain profit is a nucleolus selection. This seems especially true for cases where there are more restrictions to collaboration, which is often the case in supply chain collaboration.

\section{References}

J. F. Audy, S. D'Amours, and M. Rönnqvist. An empirical study on coalition formation and cost/savings allocation. International Journal of Production Economics, 136(1):13-27, 2012. doi: 10.1016/j.ijpe.2011.08.027.

R. J. Aumann and M. Maschler. The bargaining set for cooperative games. In H. Dresher, L. Shapley, and A. Tucker, editors, Advances in game theory, pages 443-476. Princeton University Press, Princeton, 1961.

Y. Aviv. The effect of collaborative forecasting on supply chain performance. Management Science, 47(10):1326-1343, 2001. doi: 10.1287/mnsc.47.10.1326.10260.

F. Basso, S. D'Amours, M. Rönnqvist, and A. Weintraub. A survey on obstacles and difficulties of practical implementation of horizontal collaboration in logistics. International Transactions in Operational Research, 26(3):775-793, 2019. doi: 10.1111/itor.12577.

G. R. Bitran, S. Gurumurthi, and S. L. Sam. The need for third-party coordination in supply chain governance. MIT Sloan Management Review, 48(3), 2007.

M. Christopher. Logistics \& supply chain management. Pearson, Harlow, 4 edition, 2011.

M. Dror and B. C. Hartman. Survey of cooperative inventory games and extensions. Journal of the Operational Research Society, 62(4):565-580, 2011. doi: 10.1057/jors.2010.65.

A. Elomri, A. Ghaffari, Z. Jemai, and Y. Dallery. Coalition formation and cost allocation for joint replenishment systems. Production and Operations Management, 21(6):1015-1027, 2012. doi: 10.1111/j.1937-5956.2012.01333.x. 
S. E. Fawcett, A. M. Fawcett, B. J. Watson, and G. M. Magnan. Peeking inside the black box: Toward an understanding of supply chain collaboration dynamics. Journal of Supply Chain Management, 48(1):44-72, 2012. doi: 10.1111/j.1745-493X.2011.03241.x.

W. Ferrell, K. Ellis, P. Kaminsky, and C. Rainwater. Horizontal collaboration: opportunities for improved logistics planning. International Journal of Production Research, 58(14):4267-4284, 2020. doi: 10.1080/00207543.2019.1651457.

M. Frisk, M. Göthe-Lundgren, K. Jörnsten, and M. Rönnqvist. Cost allocation in collaborative forest transportation. European Journal of Operational Research, 205(2):448-458, 2010. doi: 10.1016/j.ejor.2010.01.015.

F. Fulconis and G. Paché. Supply Chain Monitoring: LLPs and 4PL Providers as Orchestrators. Procedia - Social and Behavioral Sciences, 238:9-18, 2018. doi: 10.1016/j.sbspro.2018.03.002.

M. Guajardo and M. Rönnqvist. Operations research models for coalition structure in collaborative logistics. European Journal of Operational Research, 240(1):147-159, 2015. doi: 10.1016/j.ejor.2014.06.015.

M. Hingley, A. Lindgreen, D. Grant, and C. Kane. Using 4PL management to improve horizontal collaboration among grocery retaillers. Supply Chain Management: An International Journal, 16(5):316-327, 2011.

C. Kilger, B. Reuter, and H. Stadtler. Collaborative planning. In H. Stadtler, C. Kilger, and H. Meyr, editors, Supply Chain Management and Advanced Planning, chapter 14, pages 257277. Springer, Berlin, 5 edition, 2015.

S. Lee, C. Phalakornkule, M. M. Domach, and I. E. Grossmann. Recursive milp model for finding all the alternate optima in lp models for metabolic networks. Computers $\&$ Chemical Engineering, 24(2):711-716, 2000. doi: https://doi.org/10.1016/S0098-1354(00)00323-9.

M. Maschler. The bargaining set, kernel, and nucleolus. In R. J. Aumann and S. Hart, editors, Handbook of game theory with economic applications, volume 1, pages 591-667. 1992.

J. Nimmy, A. Chilkapure, and V. M. Pillai. Literature review on supply chain collaboration: comparison of various collaborative techniques. Journal of Advances in Management Research, 16(4):537-562, 2019. doi: 10.1108/JAMR-10-2018-0087.

G. Owen. On the core of linear production games. Mathematical Programming, 9(1):358-370, 1975. doi: 10.1007/BF01681356.

S. Pan, D. Trentesaux, E. Ballot, and G. Q. Huang. Horizontal collaborative transport: survey of solutions and practical implementation issues. International Journal of Production Research, pages 1-22, 2019. doi: 10.1080/00207543.2019.1574040.

J. A. Potters and S. H. Tijs. The nucleolus of a matrix game and other nucleoli. Mathematics of Operations Research, 17(1):164-174, 1992.

M. Raweewan and W. G. Ferrell. Information sharing in supply chain collaboration. Computers and Industrial Engineering, 126(August 2017):269-281, 2018. doi: 10.1016/j.cie.2018.09.042.

D. Schmeidler. The Nucleolus of a Characteristic Function Game. SIAM Journal on Applied Mathematics, 17(6):1163-1170, 1969.

C. Schmoltzi and C. M. Wallenburg. Operational Governance in Horizontal Cooperations of Logistics Service Providers: Performance Effects and the Moderating Role of Cooperation Complexity. Journal of Supply Chain Management, 48(2):53-74, 2012. 
C. Schneeweiss, K. Zimmer, and M. Zimmermann. The design of contracts to coordinate operational interdependencies within the supply chain. International Journal of Production Economics, 92(1):43-59, 2004. doi: 10.1016/j.ijpe.2003.10.005.

H.-J. Schramm, C. N. Czaja, M. Dittrich, and M. Mentschel. Current Advancements of and Future Developments for Fourth Party Logistics in a Digital Future. Logistics, 3(1):7, 2019. doi: $10.3390 / \operatorname{logistics} 3010007$.

L. S. Shapley. A value for $n$-person games. volume II, pages 307-317. Princeton University Press, Princeton, 1953.

D. Simchi-Levi, P. Kaminsky, and E. Simchi-Levi. Designing and Managing the Supply Chain: Concepts, Strategies, and Case Studies. McGraw-Hill, New York, 3 edition, 2008.

C. A. Soosay and P. Hyland. A decade of supply chain collaboration and directions for future research. Supply Chain Management, 20(6):613-630, 2015. doi: 10.1108/SCM-06-2015-0217.

C. Stephens. Enablers and inhibitors to horizontal collaboration between competitors: an investigation in UK retail supply chains. PhD thesis, Cranfield University, 2006.

G. C. Stevens and M. Johnson. Integrating the Supply Chain ... 25 years on. International Journal of Physical Distribution \& Logistics Management, 46(1):19-42, 2016. doi: 10.1108/ IJPDLM-07-2015-0175.

P. Sudhölter and J. A. Potters. The semireactive bargaining set of a cooperative game. International Journal of Game Theory, 30(1):117-139, 2001. doi: 10.1007/s001820100068.

J. R. G. Van Gellekom, J. A. M. Potters, J. H. Reijnierse, M. C. Engel, and S. H. Tijs. Characterization of the Owen Set of Linear Production Processes. Games and Economic Behavior, 32(1):139-156, 2000. doi: 10.1006/game.1999.0758.

S. Verstrepen, M. Cools, F. Cruijssen, and W. Dullaert. A dynamic framework for managing horizontal cooperation in logistics. International Journal of Logistics Systems and Management, 5(3-4):228-248, 2009. doi: 10.1504/IJLSM.2009.022497.

A. Win. The value a $4 \mathrm{PL}$ provider can contribute to an organisation. International Journal of Physical Distribution and Logistics Management, 38(9):674-684, 2008. doi: 10.1108/ 09600030810925962.

Z. G. Zacharia, N. R. Sanders, and N. W. Nix. The emerging role of the third-party logistics provider (3PL) as an orchestrator. Journal of Business Logistics, 32(1):40-54, 2011. doi: 10.1111/j.2158-1592.2011.01004.x. 